\section{Facilitators and barriers to advance care planning programmes targeting older care home residents: a qualitative study}

Bobo Hi-Po Lau ${ }^{1}$, PhD, James KH Luk ${ }^{2}$, FHKCP, FHKAM (Medicine), Candy HC Fong ${ }^{3}$, PhD, Amy YM Chow ${ }^{3,4}$, PhD, RSW, Cecilia LW Chan ${ }^{3,4}$, PhD, RSW, Wing Chun Ng${ }^{5}$, MBA (HSM), MN, Wai Kwong Chan ${ }^{5}, \mathrm{RN}, \mathrm{MN}$, Felix HW Chan', FHKCP, FHKAM (Medicine)

\begin{abstract}
Background. Advance care planning (ACP) is considered to be an integral part of end-of-life care. This study explored the facilitators and barriers of ACP by consolidating the practice experiences of a group of healthcare professionals involved in an outreach end-of-life programme for older people living residential care homes for the elderly (RCHEs).

Methods: Fourteen healthcare professionals participated in this qualitative study. They first completed a questionnaire to capture their views on ACP by describing a successful case. Then they took part in a focus group to discuss desired outcomes, facilitators, and barriers of ACP. Thematic analysis was applied to the questionnaire and focus group data.
\end{abstract}

Results: Participants considered ACP a valuable opportunity for older people to express their care preferences. The resultant care plan provides families and care teams a useful reference for treatment decision-making. Facilitators included the collaborative ties among the community geriatric assessment team, RCHE staff, and the families of patients; dedicated and trained staff; and well-informed family members. Barriers included delayed referral, a lack of public acceptance and awareness, and overwhelming routines among healthcare professionals.

Conclusions: Our findings underscore the multitude of individual, family, staff, and social factors affecting the dynamic process of ACP. An effective collaborative framework and professional training in communicating with distressed families are required. In addition, enhancing public awareness of comfort care and ACP is crucial.

Key words: Advance care planning; Communication; Decision making; Residential facilities; Terminal care

\section{ORIGINAL ARTICLE}

\footnotetext{
Department of Counselling and Psychology, Hong Kong Shue Yan University, Hong Kong

2 Department of Medicine and Geriatrics, TWGHs Fung Yiu King Hospital, Hong Kong

3 Department of Social Work and Social Administration, University of Hong Kong, Hong Kong

4 Jockey Club End-of-Life Community Care Project, Faculty of Social Sciences, University of Hong Kong, Hong Kong

5 Department of Community Geriatric Assessment Service, Community Care Service, Hong Kong West Cluster, Hong Kong

6 Department of Medicine, University of Hong Kong, Hong Kong
}

Correspondence to: Dr James KH Luk, Department of Medicine and Geriatrics, TWGHs Fung Yiu King Hospital, Hong Kong.Email: lukkh@ha.org.hk

\section{INTRODUCTION}

Advance care planning (ACP) facilitates adults of any age and stage of health in understanding and exchanging their personal values and goals in shaping their future care preferences. The core process involves communications between patients, family members, and healthcare providers. ${ }^{1}$ In Hong Kong, ACP is usually considered only for patients with advanced progressive diseases but is not 
restricted to mentally competent patients. When patients are incapable of expressing their views, $\mathrm{ACP}$ is discussed based on the family's knowledge of the patients' values and preferences, and the professional knowledge of healthcare providers about the patient's best interests. ${ }^{2}$ ACP has led to greater expression of wishes, concordance between preferred and delivered care, and quality of dying among the patients, as well as reduced anxiety, less depression, and lower incidence of post-traumatic stress among the family members. ${ }^{3-5}$ A systematic review of 18 studies found no evidence that ACP increased healthcare costs, and that ACP might be associated with lower healthcare costs for some patients in some circumstances. ${ }^{6}$ ACP usually involves dynamic psychosocial interplay between patients and their family members, and between families and the healthcare system. Therefore, successful discussion depends on whether the process meets socio-cultural expectations, especially those about healthcare decision-making and death. Summarising 15 studies, Lee et $\mathrm{al}^{7}$ found that, instead of individualistic self-determination, Chinese families actively take part in the ACP of their patients; therefore, they recommended a family decision-making approach.

Patients with advanced progressive diseases tend to prefer to stay in familiar places, such as their own home or RCHE. ${ }^{8}$ Place of care has been identified as one of the four key areas for improvement in the Hospital Authority Strategic Service Framework for Palliative Care. ${ }^{9}$ In October 2015, the Hospital Authority launched a programme for enhanced community geriatric assessment team (CGAT) support for end-of-life patients in RCHEs. ${ }^{10,11}$ The Hong Kong West Cluster CGAT collaborates with the Palliative Medicine Unit of Grantham Hospital, Hong Kong, to provide services to 26 RCHEs. The programme targets residents with an incurable life-threatening illness, life expectancy of around 6 months, and preference for comfort care but are currently not served by the palliative care team. $\mathrm{ACP}$ is a key component of the programme. The programme also offers enhanced clinical support, case management by a link-nurse, and expedited admission to a geriatric step-down hospital suitable for end-of-life care to the older people, as well as training on end-of-life care and ACP to the RCHE staff by Hong Kong West Cluster CGAT and the Palliative Medicine Unit.The ACP discussion involves key family members of the patient and covers the patient's state of health and anticipated progression, values and beliefs, treatment expectations and goals, support and nursing care preferences, preferences for life-sustaining treatments, and do-not-attempt cardiopulmonary resuscitation orders. The decisions are documented on a designated form and reviewed every 3 months or when the patient or family wishes to do so. The form is also applicable to cases when only the family members are involved in the discussion (eg, for cognitively incompetent patients). For cognitively competent patients, an advance directive may also be signed.

Barriers to effective implementation of ACP programmes exist on multiple levels including workload challenges of operational context, uncertain illness trajectories and prognosis, and adherence to assessments. ${ }^{12}$ Shared decision-making further increases the complexity of implementing ACP. However, clear organisational policies, systematic documentation, and availability of specially trained healthcare providers have been identified as facilitators. ${ }^{12}$

Most of the literature on ACP is from Western countries; there is insufficient understanding on what might enable or impede ACP implementation in nursing home settings in Asian populations. A study of older people living in RCHEs in Hong Kong found that $88 \%$ of participants regarded having advance directives as a good idea after receiving an introduction from healthcare professionals. ${ }^{13}$ Such a finding demonstrates that ACP could be a culturally compatible concept among residents of RCHEs and underscores the need to examine ways to incorporate $\mathrm{ACP}$ into routine residential care. This study therefore examined the facilitators and barriers to ACP for frail older people living in RCHEs by consolidating the practice experiences of professionals involved in the abovementioned programme.

\section{METHODS}

This descriptive cross-sectional qualitative study was approved by the Institutional Review Board of the University of Hong Kong / Hospital Authority Hong Kong West Cluster (Reference: UW 16-548).

Purposeful sampling was applied. All participants were professionals involved in the CGAT and RCHEs 
collaboration project who had experiences in ACP discussion with the older people and their families. Geriatricians, nurses, and nursing-home-in-charges with a variation in sex, years of service in the current position, and years of service in end-of-life care were included. Fourteen participants were recruited

\section{(TABLE I).}

Verbal consent was first sought by the study site manager. Upon receiving the verbal consent, a link to a brief introduction of the study and an online questionnaire was offered. Written informed consent was obtained before the focus group interview.
Data were collected through the self-administered questionnaire and the focus groups in December 2016.

The questionnaire was in Chinese and drafted by the multi-disciplinary research team with the purpose to orient and prepare participants for the later focus group and to collect detailed, personal views about ACP. Through open-ended questions, participants described ACP in their own words and recalled a case that they considered an example of successful ACP, explaining the factors that contributed to the success (TABLE 2). Participants were asked to complete the

TABLE 1

Characteristics of participants

\begin{tabular}{lllcc}
\hline Participant & Sex & Profession & $\begin{array}{c}\text { Duration of service in the } \\
\text { current department, } y\end{array}$ & $\begin{array}{c}\text { Duration of service in } \\
\text { end-of-life care, } y\end{array}$ \\
\hline 1 & Male & Geriatrician & 9 & 8 \\
2 & Female & Geriatrician & 16 & 7 \\
3 & Male & Geriatrician & 22 & 5 \\
4 & Female & Geriatrician & 15 & 7 \\
5 & Male & Geriatrician & 10 & 2 \\
6 & Male & Geriatrician & 17 & 7 \\
7 & Female & Nurse & 8 & 1 \\
8 & Female & Nurse & 8 & 1 \\
9 & Male & Nurse & 11 & 7 \\
10 & Female & Nurse & 7 & 3 \\
11 & Female & Nurse & 8 & 7 \\
12 & Female & Nurse & 1 & 1 \\
13 & Female & Nursing home representative & 17 & 7 \\
14 & Male & Nursing home representative & 28 & 7 \\
\hline
\end{tabular}

TABLE 2

Online self-administered questionnaire about advance care planning

Question

1. In your opinion, what is advance care planning (ACP)?

2. In your opinion, what procedures or stages does ACP involve?

3. Describe a case where you consider ACP discussion has been conducted successfully.

a. Characteristics of the patient

b. Characteristics of the family

c. Presenting problem(s)

d. Occasion and opportunity for ACP discussion

e. Strategies and skills used in discussion

f. Outcomes for the patient, the family, and the care team

g. Key to success

h. Areas of improvement

i. Key learning points

4. How much confident do you have in discussing ACP with your patients and their families? 
questionnaire at least 2 days prior to their focus group.

Focus group sessions were conducted for geriatricians and nurses separately. Each of the two sessions was a semi-structured group led by two researchers, who followed an interview guide. Participants were asked to reflect on their roles, the desired outcomes, and the facilitators and barriers to successful ACP. Participants were asked to recall their interactions with patients and their families, especially during the ACP initiation and discussion processes. Each group lasted for approximately 1.5 hours and was conducted on a weekday afternoon. The discussion was conducted and transcribed in Cantonese. Quotes included in the present paper were translated from Cantonese into English by a bilingual researcher.

Thematic analysis was applied to the qualitative data from the questionnaires and the focus groups. The analysis began with close and detailed reading of the questionnaires and the transcription. An initial coding framework was developed based on the interview guideline (roles, desired outcomes, and facilitators and barriers of $\mathrm{ACP}$ ) with themes and sub-themes constructed with reference to the literature on ACP.,12 Data were examined with respect to the coding framework. As the volume of data was modest, qualitative analysis software was not used.

\section{RESULTS}

\section{Functions and desired outcomes of ACP}

Participants reported perceiving ACP as an empowerment process for the older people to clarify and express their values and preferences, and hence, it is important that the autonomy and dignity of older people is respected throughout the ACP process. They also perceived the process as an opportunity for family members to take the perspective of the older relative, have candid conversation over the current condition, and seek various options through open discussion. Participants described the desirable outcome of ACP as a win-win situation, in which $\mathrm{ACP}$ that reflects the older person's wishes and is feasible for formal and informal caregivers to facilitate the quality of dying. The geriatrician participants emphasised that ACP is only a reference for clinical decisions and is not a legal document. Nevertheless, they regarded ACP useful for explaining aggressive high-risk treatments to patients and mitigating their fears.

There was consensus among the participants that the ACP form is primarily for documenting decisions about medical treatments. However, the consensus also acknowledged the evolving process of communication may cover psycho-socialspiritual aspects, which are important for families to fulfil unfinished businesses, funeral planning, and bereavement care. Facilitators and barriers of ACP are summarised in the Figure.

\section{Facilitators of ACP Effective collaboration}

An efficient and trustful multiparty collaboration among the geriatricians, nurses, RCHE staff, and the family members was regarded as a pivotal facilitator by the participants. They also stressed the importance of a collaborative framework that capitalises on the unique strengths of each party for effective cooperation. The geriatrician participants found themselves in an appropriate position to explain the diagnosis and prognosis, and to handle the medical-legal procedures including signing the documents, as Chinese families tend to revere doctors. In between explaining a poor prognosis and form-filling, the geriatrician participants mentioned that they relied on the nurses, who have more opportunities to meet with older people and their families, to determine psychological readiness, to elicit care wishes, to discuss care options, and to obtain consensus. The nurse participants mentioned that they relied on RCHE staff-who have more background information on the older people and their families-to identify and refer suitable cases. To facilitate close collaboration, the nurse participants suggested that RCHE staff needed to understand the value of $\mathrm{ACP}$, and thus training by the palliative care team was considered particularly helpful. The nurse participants suggested that they tended to focus on the medical aspects of care, whereas the psycho-social-spiritual needs were usually handled by RCHE staff. Lastly, families' trust of the care team, especially the RCHE staff conducting the day-to-day care of their loved ones, was considered a necessity for effective communication and decision-making by both the nurses and RCHE representatives. In summary, a tripartite collaboration is necessary to the success of ACP discussion, as mentioned by a nurse 


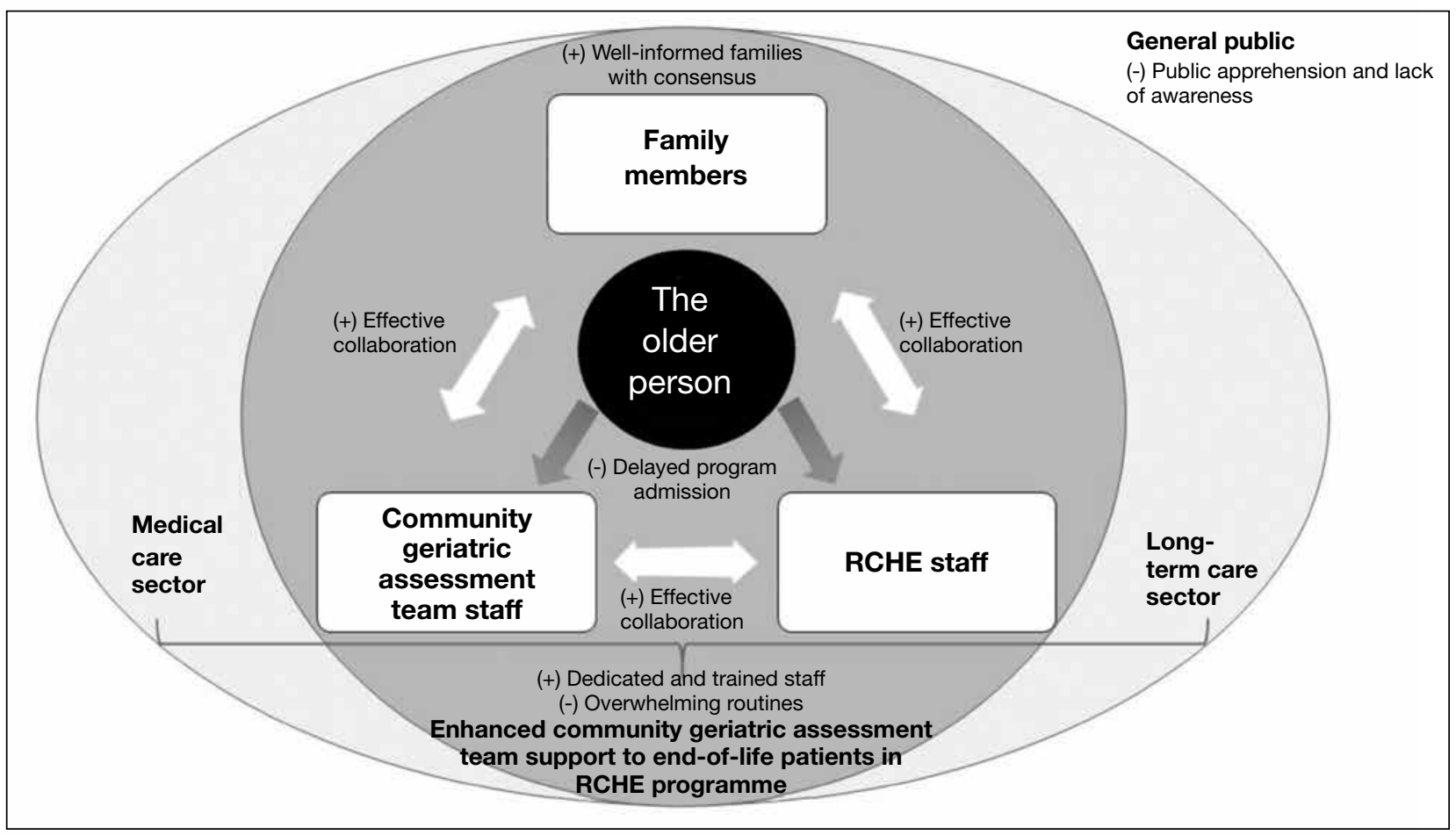

FiguRE. Facilitators (+) and barriers (-) to advance care planning implementation for older people in residential care homes for the elderly (RCHE)

participant:"There needs to be a tripartite collaboration. If we [the CGAT team], the RCHE staff and the family members can cooperate well, communicate well, and establish good rapport, things will proceed very smoothly."

\section{Dedicated and trained staff}

Trust and rapport between care staff and older people and their families was considered crucial by all participants. Hence, they suggested that competence in empathetic communication and dedication to facilitate a 'good death' were needed by all staff, as explained by a nurse participant: "My previous supervisor taught us to consider the older person in front of me as my family member when considering how to handle the case. That is, to put myself into the shoes of the distressed family."

The nurse participants remarked that skilled RCHE staff could competently provide holistic care on a daily basis and understand the value of $\mathrm{ACP}$, and act as a bridge between medical staff and older people in care and their families. They suggested reflecting on past cases through staff debriefing is useful for capacity building.

Participants remarked that open-mindedness in acknowledging possible medical futility and the familiarity of ACP in the wider healthcare system, not just the CGAT, was vital for enabling the older people and their families to trust that their plans would be actioned. Inconsistent practice may hurt public confidence and fuel apprehension, as a nurse participant mentioned: "The patient opted not to have CPR. However, when [the patient] arrived at the A\&E, the doctor conducted CPR. The family was devastated. [...] Compliance is very important, or else all effort and trust will be in vain at the very last moment."

\section{Well-informed families with consensus}

Accurate understanding about the prognosis in the family was considered key by all participants to enable candid discussion. If family members are able to visualise the suffering of their older relatives, they are more likely to understand the benefits of withdrawing futile treatments, as a geriatrician participant explained: "Every time the older person is being pushed around to receive a blood transfusion or different treatments, or is being restrained, [that person] is suffering. If the family members have seen this, they will be very willing to sign off the options".

Pictorial references of treatments (eg, nasogastric tube) were regarded as useful in educating older people and their families. Older people and their 
families may also learn from the experience of their peers. This was elaborated by a representative from RCHE: "I asked the old man, 'Look, your neighbour choked during his meal recently and he is now using a nasogastric tube. Do you want to use one if your health deteriorates?' He immediately replied with a firm 'no'. [...] I relayed his wishes to his family. [...] His daughter and wife made up their mind on this decision very quickly."

Participants agreed with the benefits of engaging the families early, ideally before their older relatives exhibit marked cognitive impairment, to allow time for those older people to inform their families of their wishes. They also opined that consensus and mutual support among key decision-makers, as well as the loving relationship between older people and their family members, renders the plan valid and acceptable to the family, avoiding family conflicts.

\section{Barriers to ACP}

\section{Delayed programme admission}

Participants remarked that some older people had missed the programme either because of difficulties in prognosis which led to delayed referral, or because they were already in severe frailty by the time the programme was implemented.

The ACP eligibility criterion regarding life expectancy is based on the Surprise Question. ${ }^{14}$ Although the geriatrician participants perceived this as a clear guideline, the nurse and RCHE representative participants remarked that frontline staff in the RCHEs found this confusing, especially in cases with dementia, general frailty, and chronic organ failure. In turn, this led to delayed referral. A RCHE representative remarked: "In some cases, we didn't think the older people would die so soon. They appeared to be in a good shape. However, they just departed at a time totally out of our expectation."

The older people were often admitted to the RCHEs with severe frailty. Participants mentioned their staff tended to find it unwelcoming to raise the issue about ACP at the first encounter of families with whom they have little rapport. More often, the families were only aware of the need for ACP after the residents' heath has deteriorated markedly or in a medical emergency. Unfortunately, in some cases, the older people died before a family consensus was reached and the documents signed.
Participants remarked that cognitive impairment limits the engagement of older people in ACP. It is therefore suggested by the geriatrician participants that, because doctors should defend the autonomy of older people, the ACP process should begin with an assessment of mental capacity.

\section{Public apprehension and lack of awareness}

Participants perceived a lack of awareness and acceptance of ACP and comfort care in the society. With limited resources, participants opined that this pilot programme could only serve families who are psychologically prepared for the discussion. Idealisation of the curative power of advanced medicine in the mass media might be to blame in hindering public acceptance toward comfort care, $\mathrm{ACP}$, and withdrawal of futile treatments, as a geriatrician participant remarked: "The media has a role, as it tends to show colourful, high-tech treatments, like heart transplantation. These could be isolated success stories. But they have not reported numerous successful cases who died in peace with comfort care".

The public need to realise that some medical procedures could be futile at best, or prolong suffering at worst, especially for frail older people. They also need to understand that comfort care does not mean relinquishing effective treatment that palliates suffering, and that requesting ACP does not imply mistrust toward the professional judgment of the clinical team.

Participants suggested that a positive attitude in society toward ACP would provide families with greater social support and ensure that they are more psychologically ready when signing the documents. In turn, this will lessen grievances. Participants thought this was also important for encouraging RCHEs and healthcare organisations to implement $\mathrm{ACP}$, and eventually feedback to an even more positive social climate. Currently, with little support from the public, ACP could easily be mistaken as a way to save healthcare cost.

Further, participants remarked that the Chinese cultural taboo over death prohibits conversation on end-of-life care and death preparation among the families. Such taboo also added apprehension among RCHE staff to promote $\mathrm{ACP}$, as a nurse participant explained:"After all, the Chinese are conservative when talking about death. Especially in private homes which 
are run for profit, if the staff talk about the prospective death of their residents with the families, doesn't it look inauspicious?"

\section{Overwhelming routines}

ACP tends to involve multiple time-consuming family meetings. Participants mentioned that the programme imposed considerable workload on already overwhelmed staff. Medical staff engage in extra hours of consultation and documentation, and the complexity of the ACP process is aggravated by logistical hiccups. Participants suggested that, in RCHEs, a favourable infrastructure was crucial to allow time for ACP-related procedures (eg, screening case files for referrals, family meetings) among other competing priorities (eg, physical care). Participants reported that high staff turnover rates led to the drainage of trained practitioners, adding difficulties to quality implementation of ACP. Participants opined that without reimbursing the additional time and manpower, RCHEs and healthcare organisations could be discouraged from the general rollout of ACP programmes.

\section{DISCUSSION}

A recent systematic review of $\mathrm{ACP}$ in nursing homes summarised five levels of facilitators, including resident, family, healthcare professional, facility, and community. ${ }^{15}$ This is consistent with our findings. That same review outlined five domains: sufficient knowledge and skills, willingness and ability to participate in $\mathrm{ACP}$, good relationship, availability of documentation system, and supportive contextual factors in the nursing home. Likewise, our findings reveal that trust and effective collaboration among the CGAT, RCHE staff and the family members, dedicated and trained healthcare professionals, and well-informed families that possess a consensus facilitates ACP. Probably, as a Chinese society, harmoniousrelationshipsamongfamilymembersand between the family and the care team are considered as a particularly important factor. However, delayed admission due to difficulties in prognostication, advanced physical and cognitive frailty of the older people, public apprehension and lack of awareness, and the already-overwhelming routine among staff were regarded as barriers. These barriers are related to the biological and organisational dimensions which are also found in Western countries. ${ }^{13,15}$ The local practice, which adopts a relatively broad definition of
ACP by including discussions with family members of cognitively incompetent older people, epitomises the importance of collective family decisionmaking. In these cases, the consensus among family members is pivotal for enabling the decision to be psychologically valid and for avoiding family conflicts during emotionally challenging times.

Van der Steen et $\mathrm{al}^{16}$ theorised that whether there is an ACP discussion depends on intrinsic factors including the willingness of the patients or their families; whereas when the discussion takes place depends on extrinsic factors such as encouragement by others (eg, the care team) or witnessing the end of someone else's life. This programme might have recruited a group of families with the right mix of intrinsic factors, including the willingness to engage in ACP with the care team. Our participants' responses further validated the importance of extrinsic factors, including the willingness of staff, as well as that of older people and their families, to engage in social learning from their peers. The right timing for initiating ACP discussion has always been a contentious issue, especially for patients with dementia. Studies have found that the worry of healthcare professionals over taking away hope from the families is a key reason for delaying ACP discussions. ${ }^{12,17}$ However, this was not revealed in our study, probably because the selected cases were often already very frail and sick. Institutionalisation, multiple hospitalisations, and marked functional decline have been recommended as appropriate timings for initiating the discussion on ACP, because the change in condition of the older people is visible to the families. ${ }^{18}$ In contrast, our participants highlighted the importance of providing empathetic support before the discussion takes place because these periods are stressful for the families.

Participants also emphasised the need for increasing awareness and understanding of comfort care and ACP, not only among the public, but also among the entire healthcare sector. To support a general rollout of the policy, professional training, frameworks, guidelines, and decision aids to facilitate communication, decision-making, and documentation are required. In addition, our findings highlight a need for promoting holistic endof-life care and death education among the general public and professionals who do not directly engage in caring for the dying. 
The present study had some limitations. Because this was a pilot programme, manpower was limited, resulting in a relatively small sample size for the study. The current study focused on how ACP was initiated and discussed from the perspective of healthcare professionals, rather than the older people and their families. Future studies may examine other aspects of the process, such as the dynamics in arriving a consensus within the families, adherence to the designated plan by medical staff outside the CGAT, and how psycho-social-spiritual care is provided in RCHEs in accordance with ACP.

About $8.5 \%$ of those aged $\geq 65$ years live in RCHEs in Hong Kong, ${ }^{19}$ and 1-year mortality can be as high as 34\% among RCHE residents with advanced dementia, ${ }^{20}$ suggesting a tremendous demand for quality end-of-life care in RCHEs. Various authorities have recommended against a routinised, "tick-box" approach, and specified ACP as a process of communication between the care team and the family. ${ }^{1}$ The current programme was an opportunity to explore the factors that influence implementation of ACP. Our findings could be useful for informing adjustments to the ACP programme before wider rollout.

\section{DECLARATION}

The authors have no conflict of interest to disclose.

\section{REFERENCES}

1. Sudore RL, Lum HD, You JJ, Hanson LC, Meier DE, Pantilat SZ, et al. Defining advance care planning for adults: a consensus definition from a multidisciplinary Delphi panel. J Pain Symptom Manage 2017;53:821-32. Crossref

2. Hospital Authority. HA Guidelines on Do-not-attempt Cardiopulmonary Resuscitation. Available from http://www. ha.org.hk/haho/ho/psrm/CEC-GE-6_en.pdf. Accessed 20 January 2016.

3. Houben CHM, Spruit MA, Groenen MTJ, Wouters EFM, Janssen DJA. Efficacy of advance care planning: a systematic review and meta-analysis. J Am Med Dir Assoc 2014;15:477-89. Crossref

4. Detering KM, Hancock AD, Reade MC, Silvester W. The impact of advance care planning on end of life care in elderly patients: randomised controlled trial. BMJ 2010;340:c1345. Crossref

5. Vandervoort A, Houttekier D, Van den Block L, van der Steen
JT, Vander Stichele R, Deliens L. Advance care planning and physician orders in nursing home residents with dementia: a nationwide retrospective study among professional caregivers and relatives. J Pain Symptom Manage 2014;47:245-56. Crossref

6. Dixon J, Matosevic T, Knapp M. The economic evidence for advance care planning: systematic review of evidence. Palliat Med 2015;29:869-84. Crossref

7. Lee MC, Hinderer KA, Kehl KA. A systematic review of advance directives and advance care planning in Chinese people from Eastern and Western cultures. J Hosp Palliat Nurs 2014;16:7585. Crossref

8. Chung RY, Wong EL, Kiang N, Chau PY, Lau JYC, Wong SY, et al. Knowledge, attitudes, and preferences of advance decisions, end-of-life care, and place of care and death in Hong Kong. A population-based telephone survey of 1067 adults. J Am Med Dir Assoc 2017;18:367. Crossref

9. Hospital Authority. The Hospital Authority Strategic Service Framework for Palliative Care. Hong Kong: The Hospital Authority; 2017

10. Luk JKH, Chan FHW. End-of-life care for advanced dementia patients in residential care home: a Hong Kong perspective. Ann Palliat Med 2018;7:359-64. Crossref

11. Luk JK. End-of-life services for older people in residential care homes in Hong Kong. Hong Kong Med J 2018;24:63-7. Crossref

12. Lund S, Richardson A, May C. Barriers to advance care planning at the end of life: an explanatory systematic review of implementation studies. PLoS One 2015;10:e0116629. Crossref

13. Chu LW, McGhee SM, Luk JK, Kwok T, Hui E, Chiu PK, et al. Advance directive and preference of old age home residents for community model of end-of-life care in Hong Kong. Hong Kong Med J 2011;17(3 Suppl 3):13-5.

14. Weissman DE, Meier DE. Identifying patients in need of a palliative care assessment in the hospital setting: a consensus report from the Centre to Advance Palliative Care. J Palliat Med 2011:14:17-23. Crossref

15. Gilissen J, Pivodic L, Smets T, Gastmans C, Vander Stichele R, Deliens L, et al. Preconditions for successful advance care planning in nursing homes: a systematic review. Intl J Nurs Stud 2017;66:47-59. Crossref

16. van der Steen JT, van Soest-Poortvliet MC, Hallie-Heierman M, Onwuteaka-Philipsen BD, Deliens L, de Boer ME, et al. Factors associated with initiation of advance care planning in dementia: a systematic review. J Alzheimers Dis 2014;40:743-57. Crossref

17. Almack K, Cox K, Moghaddam N, Pollock K, Seymour J. After you: conversations between patients and healthcare professionals in planning for end of life care. BMC Palliat Care 2012;11:15. Crossref

18. van Soest-Poortvliet MC, van der Steen JT, Gutschow G, Deliens L, Onwuteaka-Philipsen BD, de Vet HC, et al. Advance care planning in nursing home patients with dementia: a qualitative interview study among family and professional caregivers. J Am Med Dir Assoc 2015;16:979-89. Crossref

19. Luk JK, Chiu PK, Chu LW. Factors affecting institutionalization in older Hong Kong Chinese patients after recovery from acute medical illnesses. Arch Gerontol Geriat 2009;49:e110-4. Crossref

20. Luk JK, Chan WK, Ng WC, Chiu PK, Ho C, Chan TC, et al. Mortality and health services utilisation among older people with advanced cognitive impairment living in residential care homes. Hong Kong Med J 2013;19:518-24. Crossref 